\title{
Highly Tunable Aptasensing Microarrays with Graphene Oxide Multilayers
}

SUBJECT AREAS:

BIOSENSORS

OPTICAL PROPERTIES AND

DEVICES

DESIGN, SYNTHESIS AND

PROCESSING

Received

26 September 2013

Accepted

13 November 2013

Published

28 November 2013

Correspondence and requests for materials should be addressed to

B.-S.K. (bskim 19@ unist.ac.kr)
Yun Kyung Jung, Taemin Lee, Eeseul Shin \& Byeong-Su Kim

Interdisciplinary School of Green Energy, Ulsan National Institute of Science and Technology (UNIST), UNIST-gil 50, Eonyang-eup, Ulju-gun, Ulsan 689-798, Republic of Korea.

A highly tunable layer-by-layer (LbL)-assembled graphene oxide (GO) array has been devised for high-throughput multiplex protein sensing. In this array, the fluorescence of different target-bound aptamers labeled with dye is efficiently quenched by GO through fluorescence resonance energy transfer (FRET), and simultaneous multiplex target detection is performed by recovering the quenched fluorescence caused by specific binding between an aptamer and a protein. Thin GO films consisting of 10 bilayers displayed a high quenching ability, yielding over $85 \%$ fluorescence quenching with the addition of a $2 \mu \mathrm{M}$ dye-labeled aptamer. The limit for human thrombin detection in the 6- and 10-bilayered GO array is estimated to be 0.1 and $0.001 \mathrm{nM}$, respectively, indicating highly tunable nature of LbL assembled GO multilayers in controlling the sensitivity of graphene-based FRET aptasensor. Furthermore, the GO chip could be reused up to four times simply by cleaning it with distilled water.

ver since the first successful isolation, graphene and related structures have emerged as promising nano-
materials for diverse applications because of their remarkable mechanical, electronic, thermal, and optical
properties. Of these different structures, graphene oxide (GO) composed of both conjugated $\mathrm{sp}^{2}$-systems and electronically isolated oxygen functional groups possesses unique characteristics, such as a high aqueous dispersibility, easy synthetic functionalization, high biocompatibility, and photoluminescence ${ }^{1-5}$. In particular, the heterogeneous chemical, atomic, and electronic structures of GO cause it to retain near-infrared, visible, and ultraviolet fluorescence ${ }^{1,5}$. Moreover, the graphitic carbon of GO allows the quenching of nearby fluorescent species, such as dyes ${ }^{6}$, conjugated polymers ${ }^{7}$, and quantum dots ${ }^{8}$, by both long-range energy-transfer and electron-transfer processes ${ }^{9-11}$. Based on these unique optical properties, many solution-based sensors employing fluorescence quenching based on fluorescence resonance energy transfer (FRET) ${ }^{1-3}$ or non-radiative dipoledipole coupling ${ }^{1,6-8}$ have been exploited for the homogeneous detection of DNA ${ }^{11-17}$, proteins ${ }^{8,18-23}$, enzymes ${ }^{24-29}$, metal ions ${ }^{30-33}$, and cancer cells ${ }^{34}$.

Ionic groups and aromatic domains in GO allow it to bind dye-labeled single-stranded DNAs (ssDNAs) and charged proteins via electrostatic and $\pi-\pi$ stacking interactions, resulting in the quenching of adjacent dyes ${ }^{1-3,11-33}$. However, in the presence of the target analyte, the competitive binding of the target molecule for dye-labeled probes allows desorption of probes from the GO surface, leading to recovery of the initially quenched fluorescence.

Aptamers selected by in vitro selection have been in the limelight as affinity probes due to their high affinity, stability, and specificity for a wide range of targets, including DNA, proteins, and toxic substances ${ }^{34-36}$. Using aptamers as probes, bovine thrombin detection was performed with fluorescence quenching by incubating 6carboxyfluorescein (FAM)-labeled thrombin aptamers and graphene, and fluorescence recovery was induced by a subsequent thrombin addition ${ }^{12,18}$. This recovery is mainly explained by the quadruplex structure of aptamers formed by thrombin, which has a weak affinity to graphene. This aptamer is uniquely appropriate for graphenebased FRET biosensor studies as an ideal recognizing element. However, the solution FRET sensor made of aptamers is not suitable for high-throughput and multiplexed analysis. Microarray technology has become a common analytical tool for biochemical analysis because of its intrinsic advantages in high-throughput monitoring, multiplex biochemical detection, miniaturization, small reaction volumes, low-manufacturing costs, simplicity, robustness, and parallel analysis for many samples of interests in a single slide ${ }^{37-41}$. Furthermore, a glass surface, when appropriately treated, exhibits very low non-specific attachment to labeled probes, resulting in lower backgrounds ${ }^{41}$. These features provide a significantly improved efficiency that is unattainable with other methods.

DNA microarrays are typically printed robotically onto a glass microscope slide. However, since predominantly micrometer-sized GO is too large to be spotted by microarray spotter as well as has planar structure, the spotting using a microarray spotter is challenging. For example, Seo and coworkers used a simple dropping 
method to fabricate GO arrays ${ }^{13,30}$. Although they successfully detected specific DNA hybridization and heavy metal ions, the GO film deposited on the glass slide by pipette dropping cannot control surface adhesive strength, charges, or thickness. Ultimately, the intensity of a false green color exhibited by GOs under $532 \mathrm{~nm}$ excitation $\left(\lambda_{\mathrm{em}}=559-610 \mathrm{~nm}\right)$ often limits its application in making reproducible GO chip sensors.

A number of approaches have been reported to assemble the GO nanosheets into thin films with tailor-made properties. Among these approaches, LbL assembly has been adopted for the immobilization of GO on solid glass substrates ${ }^{42-47}$. This technique is a versatile, highly tunable, and scalable approach to forming thin films via alternating adsorption of positively and negatively charged species on a given substrate. Furthermore, the thickness of the LbL multilayer GO thin films can be precisely controllable at a subnanometer level simply by varying the number of stacking layers. Because the GO film thickness is closely correlated with its optical and electrical properties, the technique of fabricating the GO films with controllable supramolecular structure is highly critical to enhance the fluorescence on/off signal ratio.

Herein, we report a GO multilayer aptasensor array in which the fluorescence quenching of dye-labeled aptamers with different sequences is observed and multiplex target detection is performed by making an optical improvement in the quenched fluorescence. Using a silicon isolator/glass hybrid chip system, a regular array of graphene sheets was generated at a defined location with desired patterns on a microscope slide, which is suitable for the rational design of GO arrays. Our GO sensor is more robust than a typical DNA microarray and is even recyclable, giving it another promising merit in array systems.

\section{Results}

Preparation of a GO array for the detection of target protein. The scheme for preparing LbL-assembled GO multilayer arrays and aptamer-based protein sensing on a GO multilayer is illustrated in Figure 1. Initial GO suspensions prepared using a modified Hummers method $^{48,49}$ have carboxylic acid $(\mathrm{COOH})$ groups conferring negative charges $\left(\mathrm{GO}^{-}\right)$over a wide $\mathrm{pH}$ range. Positively charged $\mathrm{GO}\left(\mathrm{GO}^{+}\right)$ sheets were produced by introducing amine $\left(\mathrm{NH}_{2}\right)$ groups of ethylenediamine via the 1-ethyl-3-(3-dimethyl aminopropyl)-1carbodiimide hydrochloride (EDC)-mediated coupling reaction. $\mathrm{GO}^{-}$sheets were successfully self-assembled on a positively charged amine-glass slide by a strong electrostatic attractive force, preventing the detachment of GO sheets during extensive washing cycles. Subsequently, a regular array of multilayer thin films was assembled by repeatedly layering the $\mathrm{GO}^{+}$and $\mathrm{GO}^{-}$suspensions at a defined location to afford a multilayer buildup of $\left(\mathrm{GO}^{+} / \mathrm{GO}^{-}\right)_{n}(n=$ number of bilayers, BLs) (Figure 1a), which is a new design for GO arrays in biosensing applications. We chose a $\mathrm{pH}$ of 3 for dip-coated $\mathrm{LbL}$ assemblies of both $\mathrm{GO}^{-}$and $\mathrm{GO}^{+}$with an optimum colloidal stability and sufficient surface charges. We chose this $\mathrm{pH}$ because non-homogeneous GO films were produced when we used $\mathrm{GO}^{-}$ solution at $\mathrm{pH} 4$ and $\mathrm{GO}^{+}$solution at $\mathrm{pH} 11$ for LbL assembly (Figure S1a and Figure S1b). Additionally, when the GO array was thermally reduced at 150,300 , and $450^{\circ} \mathrm{C}$ for $30 \mathrm{~min}$ in order to decrease the green signal intensities of GO spots and remove electronically isolated oxygen functional groups such as hydroxyl and carboxyl moieties, background signals outside GO spots were stood out (Figure S1c). These non-homogeneous films produced strong, nonspecific fluorescence signals, ultimately lowering the specificity of target detection (Figure S2).

The efficient construction of fluorescent aptasensors requires the controllable proximity of dyes to the graphene surface and obvious fluorescence changes induced by targets. As shown in Figure 1b, aptamer-graphene binding guarantees the close proximity of dyes to graphene and the following FRET from FAM dyes to graphene results in the highly efficient fluorescence quenching of the dyes. More importantly, the conformation of aptamers on graphene can a

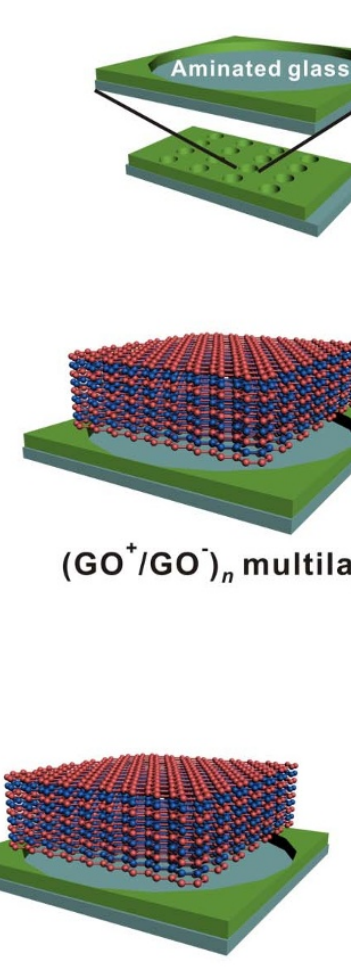

Dip-coating

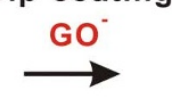

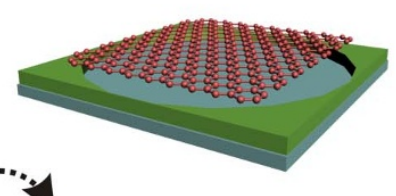

$\downarrow \underset{\mathrm{GO}^{+}}{\text {Dip-coating }}$
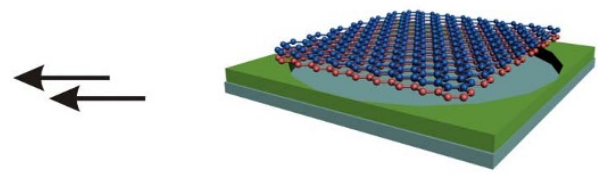
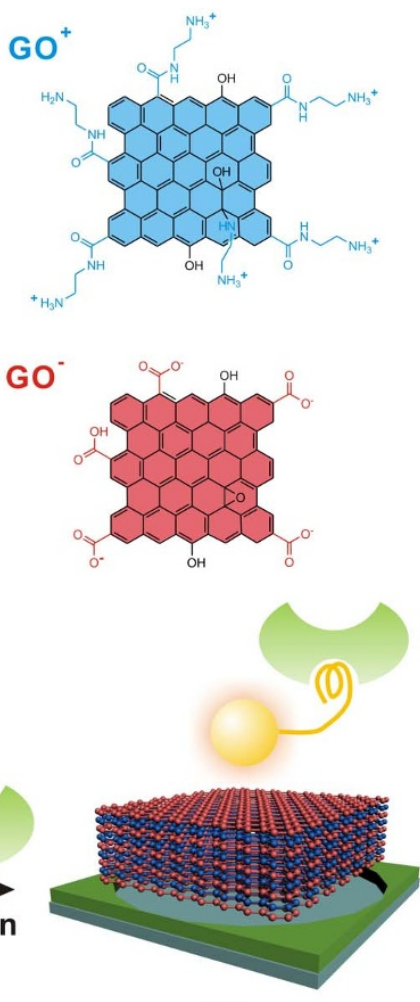

ON

Figure 1 Schematic representation of (a) the LbL-assembled GO multilayer array and (b) the aptamer-based protein sensing mechanism of the LbL GO multilayer array. 
be changed by quadruplex formation induced by the target protein, such as thrombin ${ }^{12,18}$. Weak binding between quadruplex-protein complexes and the graphene surface keeps the FAM dye away from the graphene surface, which induces fluorescence recovery in the GO multilayer array. Therefore, the final capping layer of the GO array must be $\mathrm{GO}^{-}$because the biosensing platform is noncovalently constructed only by $\pi-\pi$ stacking interactions between aptamer bases and GO. When $\mathrm{GO}^{+}$was located in the top layer instead, a FAMlabeled aptamer was not detached from the GO surface even after incubation with the target protein due to the strong electrostatic attraction between positively charged $\mathrm{GO}^{+}$and negatively charged phosphate backbones in aptamer (data not shown).

Characterization of the LbL-assembled GO multilayer array. Multilayer fabrication was monitored by the gradual increase of characteristic UV/Vis absorbance spectra (Figure 2a). The optical image in the inset of Figure $2 \mathrm{a}$ illustrates that the 10-BL GO array is morphologically homogeneous in all spots, and the array was thus used in the following fluorescence studies. The GO spectrum contains a strong absorption band at $230 \mathrm{~nm}$, which corresponds to $\pi-\pi^{*}$ transitions of $\mathrm{C}=\mathrm{C}$ bonds in $\mathrm{sp}^{2}$ hybrid regions ${ }^{5}$. Figure $2 \mathrm{~b}$ displays a linear increase in the characteristic absorbance at $230 \mathrm{~nm}$ with respect to the number of BLs. Consistent with the UV/Vis absorption spectra, the surface profiler measurement indicated that the thickness of patterned GO multilayer films is linearly proportional to the number of BLs with an average BL thickness of $4.30 \mathrm{~nm}$, which is considerably thicker than that assembled non-patterned surface $(1.6 \mathrm{~nm})$ due to owing to condensation and weak desorption of GO nanosheets inside the pattern. Figure $2 \mathrm{c}$ presents height-mode atomic force microscopy (AFM) images from 2- and 10-BL GO films. Initial 2-BL deposition displayed a smooth GO surface. As the deposition progresses to a higher number of BLs, however, the entire surface became relatively rough with an appearance of characteristic wrinkles of GO nanosheet. Surface root-mean-square roughness $\left(\mathrm{R}_{\mathrm{rms}}\right)$ values (averaged over $5 \times 5 \mu^{2}$ ) of 2- and 10-BL GO films were determined to be 2.6 and $10.7 \mathrm{~nm}$, respectively (Figure S3). From the optical and structural characterizations, the GO array created by LbL assembly is highly controllable.

Specificity and sensitivity of the GO aptasensing array. To carry out a proof-of-concept to detect individual target analytes in the GO array, $100 \mathrm{nM}$ of FAM-labeled thrombin aptamer was treated first. Although the pristine multilayered GOs displayed a weak green color in the fluorescence scanner at $530 \mathrm{~nm}$ excitation, the fluorescence of the dye was completely quenched when we compared the PL intensity before and after FAM-labeled thrombin aptamer attachment (Figure 3a). This quenching occurs as the aromatic bases in aptamer serves as acceptors as part of the FRET phenomenon. We considered the fluorescence intensities obtained after aptamer immobilization to be a criterion for detecting the target analyte. As demonstrated, after the probe DNAs on the GO surface were bound to their target thrombin, which was dissolved in HEPES buffer (1 mM HEPES, $1 \mathrm{mM} \mathrm{MgCl}_{2}, \mathrm{pH}$ 7.26), the GO fluorescence signal increased 34 times due to the detachment of FAM-labeled aptamers from the GO surface (Figure $3 \mathrm{a}$ and Figure $3 \mathrm{~b}$ ). These results indicated that the fluorescence recovery of the FAM-labeled aptamers in the presence of thrombin was caused by the high affinity between aptamers and thrombin and the subsequent formation of a quadruplex-thrombin complex. In contrast, the negative control analytes containing $100 \mathrm{nM}$ bovine serum albumin (BSA), streptavidin (STA), glucose, and human immunoglobulin G (IgG) antibody were incubated on the FAM-aptamer-treated GO spots and
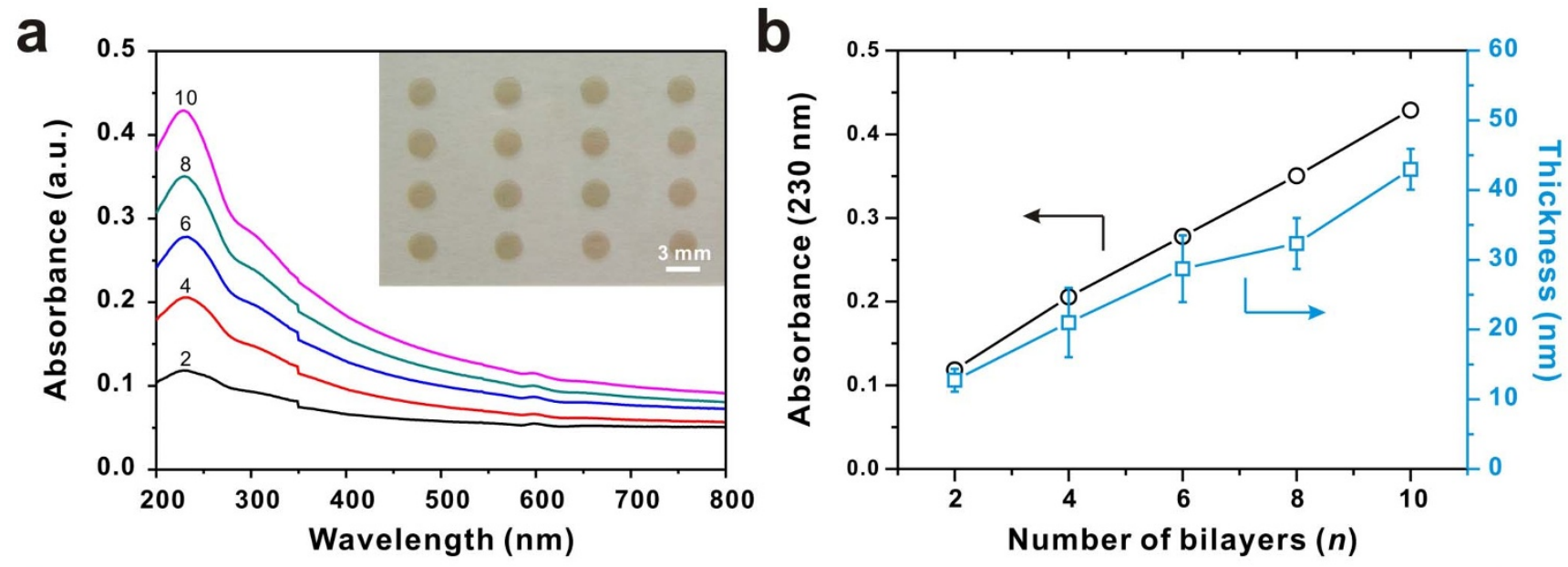

C

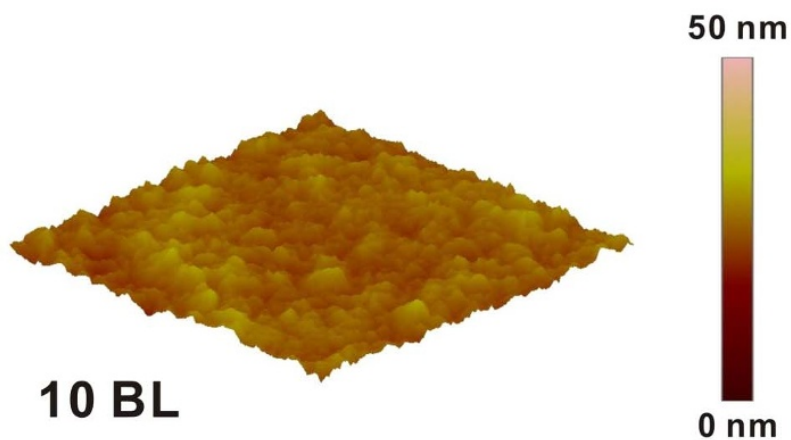

Figure $2 \mid$ (a) UV/Vis growth curve of an LbL-assembled GO multilayer. The number on the graph indicates the number of BL. Inset provides a representative image for the 10-BL GO array. (b) Corresponding absorbance and thickness with respect to the number of BL. The thickness of patterned GO multilayer was measured with a surface profiler on ten individual spots. (c) Representative height-mode AFM images of 2- and 10-BL GO multilayers $\left(5 \times 5 \mu \mathrm{m}^{2}\right)$. 
a

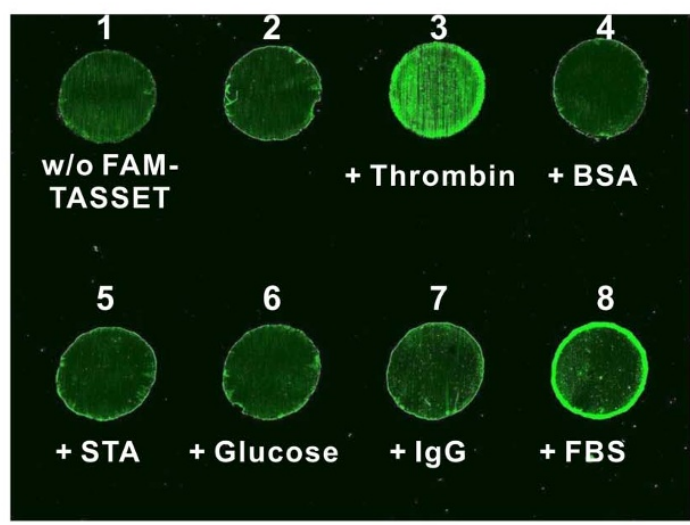

C

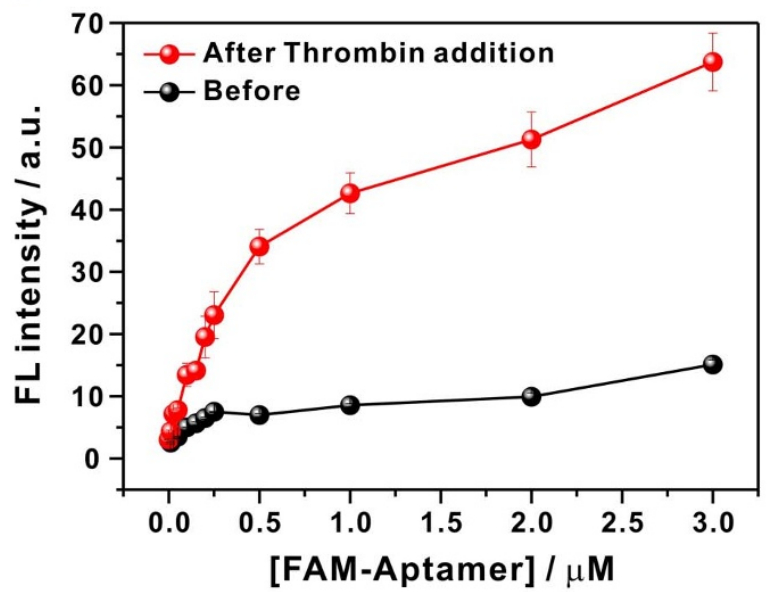

b
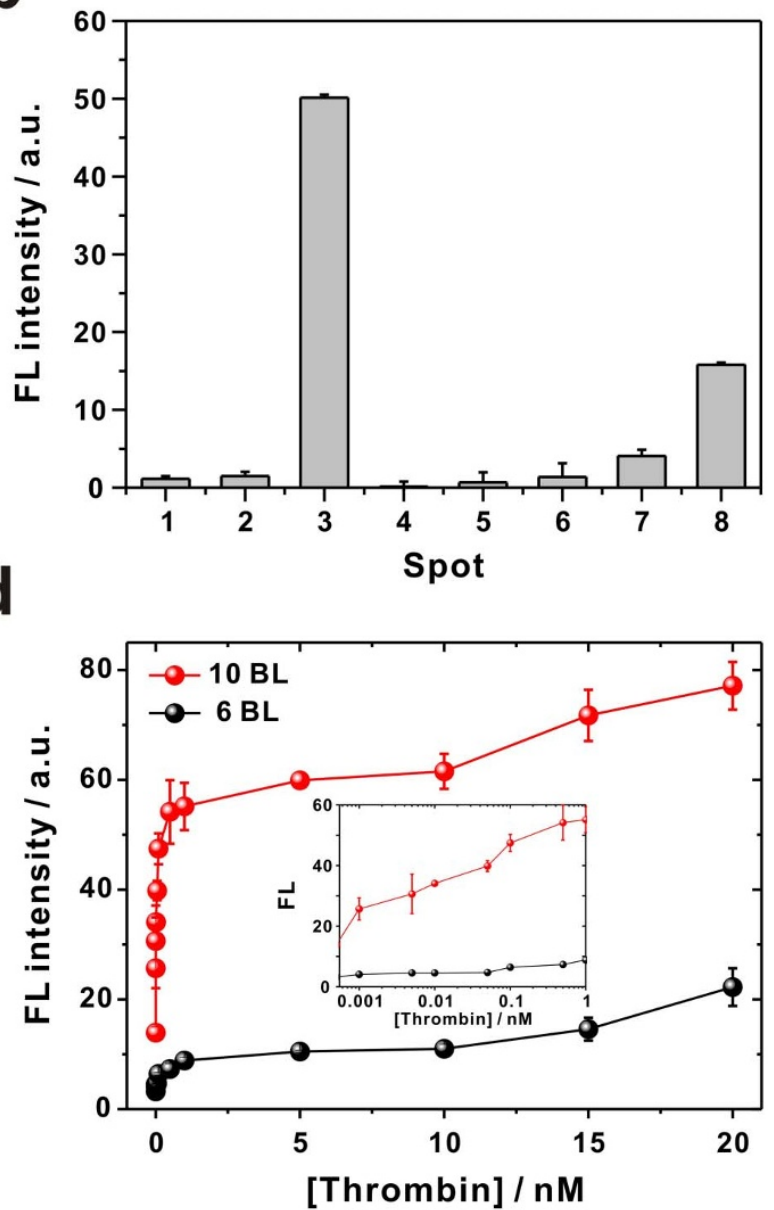

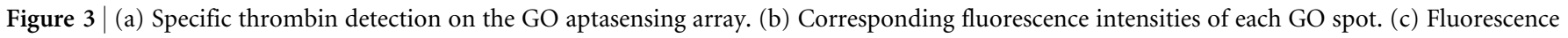

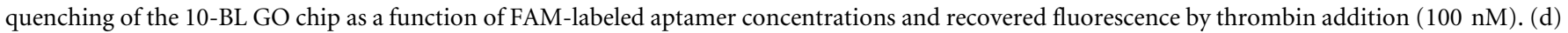
The sensitivity of target protein detection on the array according to the number of bilayers. Inset graph shows the change of fluorescence intensity in the GO array after addition of thrombin in the picomolar range.

did not have any noticeable effect on the GO fluorescence signals, further confirming the specificity of the detection. In a $1 \%$ FBStreated GO spot, however, the fluorescence was brightened only on the edge, likely due to nonspecific interactions with FBS, which contains a rich variety of proteins. These results indicate that the GO aptasensing array could selectively capture target proteins due to the high specificity of the aptamer.

To evaluate the quenching efficiency of a multilayered GO array, a wide range of FAM-labeled thrombin aptamer concentrations (from $10 \mathrm{nM}$ to $3 \mu \mathrm{M}$ ) was incubated on a 10-BL GO array (Figure $3 \mathrm{c}$ and Figure S4). Over $85 \%$ of the fluorescence was quenched within the addition of $2 \mu \mathrm{M}$ aptamer, indicating a high quenching ability by multilayer GO thin films. This highly efficient quenching is considered to be the direct consequence of a high-efficiency energy transfer between the dyes and graphene. When adding $100 \mathrm{nM}$ of thrombin to the GO array complexed with FAM-labeled aptamer, concentration-dependent fluorescence recovery was observed, as shown in Figure $3 c$ and Figure S4. The intensity of thrombin-recovered fluorescence was approximately five times as much as those without thrombin, which supports the practicality of our GO aptasensing array. Figure $3 \mathrm{~d}$ and Figure S5 illustrate the sensitivity of this array for target protein detection depending on the BL number. After loading $100 \mathrm{nM}$ of FAM-labeled aptamer at each spot of the 6- or 10-BL GO array, we applied serially diluted target proteins ranging from 0.001 to $20 \mathrm{nM}$. The fluorescence intensity of the GO aptasensor was enhanced with increasing thrombin concentrations. As the number of layers increased, the fluorescence recovery signal also became increasingly noticeable. The restored fluorescence signal of 10-BL GO array following the thrombin reaction was calculated to be five to six times higher than that of the 6-BL GO array, and the limit of detection (LOD) for thrombin in the 6- and 10-BL GO arrays was determined to be 0.1 and $0.001 \mathrm{nM}$, respectively (Figure $3 \mathrm{~d}$ ). It is of note that the LOD in the 10 -BL GO array is 30 -fold more sensitive than solution based graphene FRET aptasensor ${ }^{18}$. The higher recovery signal in the thicker GO array can be ascribed to the density of FAM-labeled aptamers adsorbed on the GO surface and the high efficiency of GO as a quencher of the FRET probe. ssDNA preferentially binds to thicker layers and to wrinkles rather than to flat GO surfaces ${ }^{2,50,51}$. Thus, the quantity of aptamers folded by thrombin could be more abundant on a thicker multilayer surface, resulting in more sensitive thrombin detection by fluorescence responses. Therefore, the highly controllable LbL assembled GO aptasensing array can afford the tunable platform for extremely sensitive protein detection. However, the 15- and 20-BL GO array did not significantly improve the detection sensitivity due to the uplift of the background under our experimental condition.

Recyclability of the GO array. Both conventional DNA microarrays and previously developed GO arrays have a single-use chip format. One of the main concerns of biochip development is recyclability. Because the immobilization of aptamers on our GO array is based on noncovalent $\pi-\pi$ stacking between nucleobases and the $\mathrm{sp}^{2}$ carbon of 
GO, it was expected that aptamers, aptamer-target complexes, or a mixture of aptamers and non-target molecules can be easily washed away from the GO sheet. We found that by soaking and rinsing used chips in distilled water, the chips were completely cleaned and could be re-used up to four times (Figure 4a).

Simultaneous detection of multiple proteins on the GO array. Encouraged by the successful individual protein detection, we extended the approach toward the detection of multiplexed proteins on a single microarray platform (Figure $4 \mathrm{~b}$ and Figure 4c). We selected aptamer-protein pairs to analyze four different proteins, and each protein-specific aptamer was labeled with FAM dye and loaded onto the designated positions of a GO array. FAM-labeled C10 composed of 10 cytosine $(\mathrm{C})$ sequences was used as a control ssDNA, and it was deposited on the remaining GO spots. When all four different proteins were added to the spots pretreated with pair aptamers as well as $\mathrm{C} 10$ spots on the same vertical line, the GO fluorescence running in a diagonal line from the upper left to the lower right was significantly enhanced (Figure $4 \mathrm{~b}$ ). On the other hand, the fluorescence from the PDGF aptamer-coated GO spot was selectively strengthened upon PDGF addition without other signals (Figure 4c). Because many DNA aptamers can reportedly engage in the specific binding of various targets, the GO aptasensing array platform can be applied to the multiplex analysis of widespread target analytes.

\section{Discussion}

From our experimental result, we could conclude that our GO array in which thickness is controlled by LbL assembly method differs in quenching degrees of fluorescent probe and requires different amount of target molecule for fluorescence restoration according to its thickness. Multilayer GO array stacked on amine glass substrate with electrostatic interactions does not detach from the glass even by harsh washing, but, fluorophore-labeled aptamers, aptamer-target complexes, or a mixture of aptamers and non-target molecules bound on GO spots by noncovalent interactions are easily removed by simple rinsing, therefore demonstrating the benefits of recyclable array platform. Newly designed GO array provides higher sensitivity to detect its target molecule than solution-based GO sensor. In addition, our homogeneous GO array having the same form with conventional DNA array chip shows practicability as well as expandability for multiplexed target detection.

In summary, we fabricated a highly controllable GO array platform using an LbL assembly method to develop a high-throughput FRET aptasensor. The GO multilayer was deposited on an amine
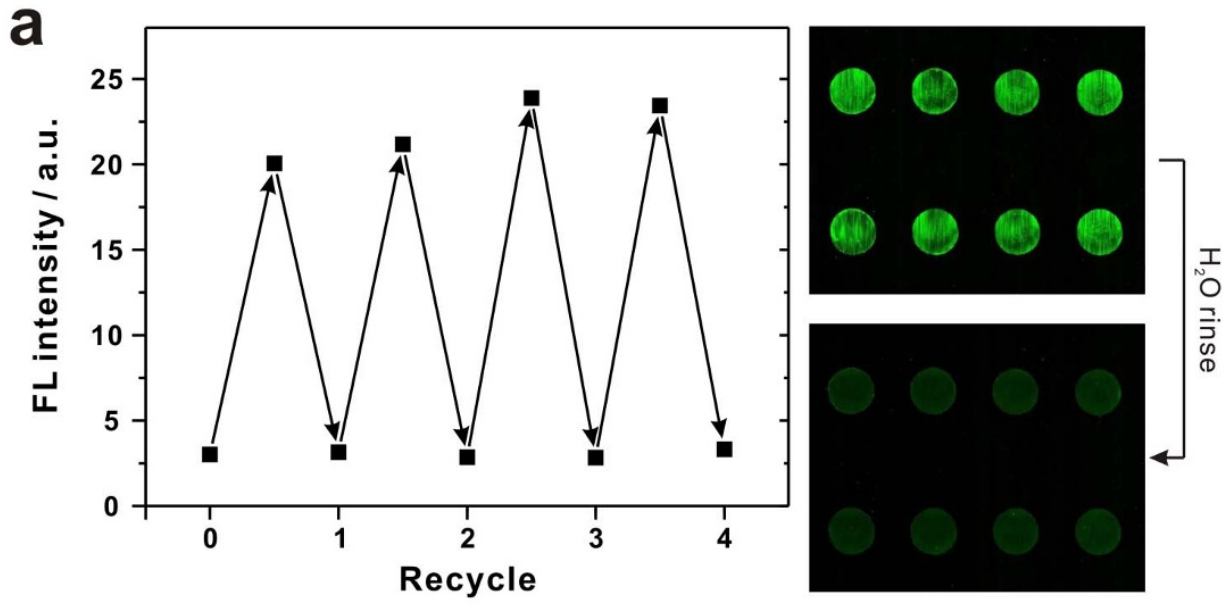

b

Thrombin PDGF VEGF Nucleolin

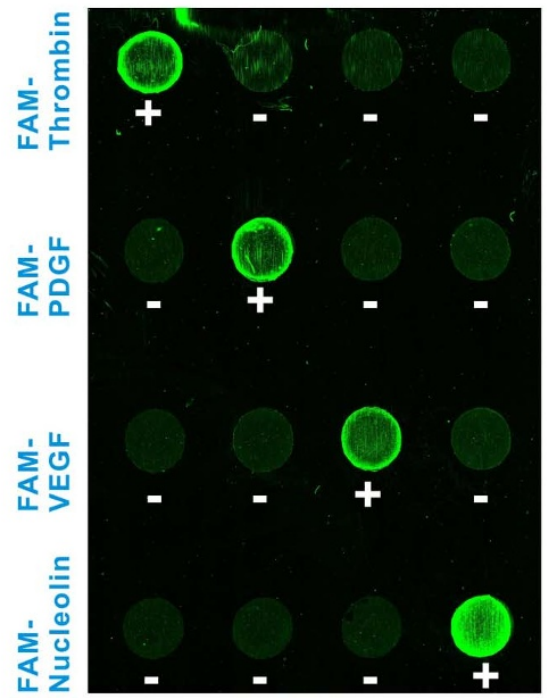

C Nucleolin PDGF Thrombin VEGF

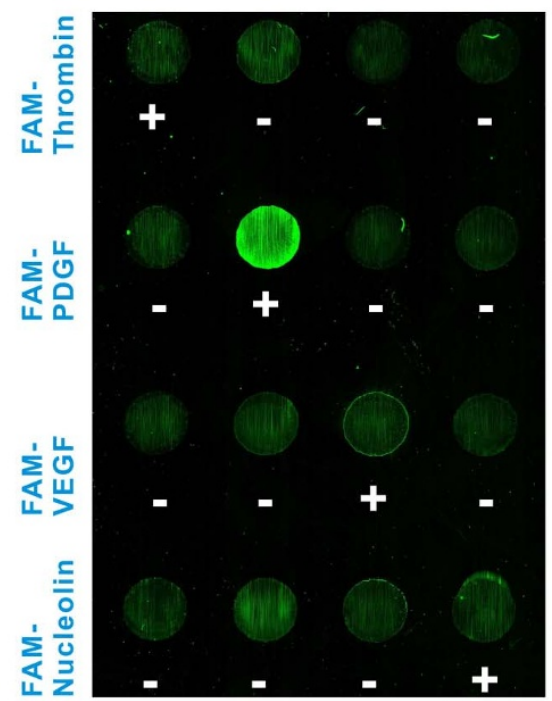

Figure $4 \mid$ (a) Recyclability of the GO array by simply cleaning with distilled water. (b) Multiplex detection of four different proteins using their FAMlabeled binding aptamers. (c) Selective fluorescence recovery of the PDGF aptamer-coated GO spot upon PDGF addition. Plus and minus sign respectively indicate the loading and unloading of both specific FAM-labeled aptamers and proteins onto the positions. 
glass slide by electrostatic interactions, and the resultant GO multilayer was engineered to have a negative charge for the noncovalent binding of the aptamer with GO. The GO aptasensor array was derived from the fluorescence quenching of FAM-labeled aptamers by GO and the subsequent fluorescence recovery induced by the formation of quadruplex-protein complexes. A higher number of GO BL films was more sensitive to detecting the target protein because of the high-density aptamer binding and efficient fluorescence quenching of GO. This GO aptasensor array can also be applied to simultaneous multiplex protein detection with a high specificity. Based on its excellent performance, the solid-phase GO aptasensor array is promising for high-throughput biological/chemical detection, and recyclable device platforms.

\section{Methods}

Materials. 6-Carboxyfluorescein (FAM)-labeled oligonucleotides were synthesized and purified by Integrated DNA Technologies (Coralville, IA, USA). Recombinant human platelet-derived growth factor (PDGF)-BB was purchased from R\&D Systems (Minneapolis, MN, USA). Nucleolin was purchased from Abcam (Cambridge, MA, USA). Fetal bovine serum (FBS) was obtained from Gibco (Grand Island, NY, USA). The other proteins and chemicals including thrombin from human plasma, bovine serum albumin (BSA), streptavidin (STA), immunoglobulin G (IgG), glucose, vascular endothelial growth factor (VEGF), 1-ethyl-3-(3-dimethylaminopropyl)-1carbodiimide hydrochloride (EDC), ethylenediamine, $\mathrm{HEPES}$, and $\mathrm{MgCl}_{2}$ were purchased from Sigma-Aldrich, Inc. (St. Louis, MO, USA) and used without further purification. Sample stock solutions were prepared by directly dissolving the proteins in HEPES buffer ( $1 \mathrm{mM}$ HEPES, $\left.1 \mathrm{mM} \mathrm{MgCl}_{2}, \mathrm{pH} 7.26\right)$ and stored in a refrigerator at $-20^{\circ} \mathrm{C}$

Preparation of negatively charged graphene oxide $\left(\mathrm{GO}^{-}\right)$and positively charged GO $\left(\mathbf{G O}^{+}\right)$. Graphite oxide was synthesized by a modified Hummers method ${ }^{48,49}$ and then exfoliated to yield a brown GO dispersion under ultrasonication. The resultant GO was negatively charged over a wide variety of $\mathrm{pHs}$ because the GO sheet contains chemical functional groups, such as carboxylic acids. $\mathrm{A} \mathrm{GO}^{+}$suspension with a dark brown color was synthesized by reacting a $\mathrm{GO}^{-}$suspension $(0.5 \mathrm{mg} / \mathrm{mL}, 200 \mathrm{~mL})$ with EDC (2.5 g) and ethylenediamine $(20 \mathrm{~mL})$ for $12 \mathrm{~h}$, and dialyzing for three days to remove excess EDC and ethylenediamine with $13 \mathrm{kDa}$ MWCO dialysis tubing (Spectra/Por dialysis membrane). $\mathrm{GO}^{+}$and $\mathrm{GO}^{-}$suspensions were adjusted to $\mathrm{pH} 3$ with $1.0 \mathrm{M} \mathrm{HCl}$

Fabrication of a GO array by layer-by-layer (LbL) assembly. A commercially available silicone isolator $(4 \times 6$ holes, $2.5 \mathrm{~mm}$ diameter $\times 0.5 \mathrm{~mm}$ depth, $25 \mathrm{~mm}$ height $\times 54 \mathrm{~mm}$ width) was cleaned and attached to an amine glass slide. The positively charged amine glass hybridized with a silicone isolator was dipped and spun in a $\mathrm{GO}^{-}$solution $(0.5 \mathrm{mg} / \mathrm{mL}, \mathrm{pH} 3)$ for $10 \mathrm{~min}$, leading to the adsorption of one monolayer and to the reversal of the surface charge. The substrate was then rinsed three times with distilled water at the same $\mathrm{pH}$ for $1 \mathrm{~min}$ to remove the loosely adsorbed GO from the substrate. Next, the substrate was coated with a $\mathrm{GO}^{+}$solution $(0.5 \mathrm{mg} / \mathrm{mL}, \mathrm{pH} 3)$, followed by a rinsing step. We then obtained a 1-BL GO sheet. The above procedures were repeated to achieve the desired number of BLs.

\section{Characterization of the LbL multilayer GO thin films. Multilayer GO thin films} were fabricated on quartz glass or silicon wafers. The transmittance of graphene films on quartz glass was characterized by UV/Vis spectroscopy (VARIAN, Cary 5000). The thickness of GO multilayers on the silicon substrate was measured by ellipsometry (J. A. Woollam Co. Inc, EC-400 and M-2000V). The average thickness values were calculated with three individual measurements. AFM images of GO multilayers coating the silicon wafer were measured in tapping mode with Nanoscope $\mathrm{V}$ from Veeco.

Detection of target proteins. For specificity studies, $5 \mu \mathrm{L}$ of FAM-labeled thrombin aptamer (100 nM, TASSET; 5' - AGTCCGTGGTAGGGCAGGTTGGGGTGACT$3^{\prime}$ ) was loaded and incubated on the GO array for $1.5 \mathrm{~h}$ on a twister shaker (FinePCR TW3, Rose Scientific Ltd, Alberta, Canada) at ambient humidity levels of 50-60\%. After incubating $5 \mu \mathrm{L}$ of thrombin $(100 \mathrm{nM})$, BSA $(100 \mathrm{nM})$, STA (100 nM), glucose (100 nM), IgG (100 nM), and FBS (1\%) in HEPES buffer (1 mM HEPES, $1 \mathrm{mM}$ $\mathrm{MgCl}_{2}, \mathrm{pH} 7.26$ ) with the as-prepared aptamer probe-loaded GO arrays for $2 \mathrm{~h}$, the fluorescence emission signals of the GO array were detected with an Agilent G2565CA microarray scanner (Agilent Technologies, Inc. Santa Clara, CA, USA) using a $532 \mathrm{~nm}$ excitation laser. A laser fluorescent scanner was used to detect the binding signals from the FAM fluorophore. The average relative fluorescence intensity of each spot was analyzed with Image J software for triplicate experiments. To investigate fluorescence quenching and recovery on the GO array according to the concentration of FAM-labeled probe aptamers, a large range of FAM-labeled aptamer concentrations $(0,10,25,50,100,150,200,250,500,1,000,2,000$, and $3,000 \mathrm{nM})$ was dropped on the GO array, and then, $100 \mathrm{nM}$ of thrombin was incubated on each spot. A fluorescence intensity curve was drawn before and after thrombin addition by Origin software. To verify the sensitivity of the GO array depending on the number of
BLs, different concentrations of thrombin $(0,0.001,0.005,0.01,0.05,0.1,0.5,1,5,10$, 15 , and $20 \mathrm{nM}$ ) were added to 6- or 10-BL GO arrays treated with $100 \mathrm{nM}$ of FAMlabeled aptamer.

Recyclable GO array. After sequential reactions of aptamer probes labeled with a green fluorophore, FAM and target proteins, a GO array chip was cleaned with distilled water to eliminate the aptamer-protein complex. The reusability of the GO array chip was analyzed by repeatedly using the chip.

Multiplexed detection of various target proteins. Target binding partners (aptamers) labeled with FAM were prepared to simultaneously detect various protein targets, including thrombin, PDGF, VEGF, and nucleolin, in one GO array chip. The sequences of the aptamer were $5^{\prime}$-CAGGCTACGGCACGTAGAGCATCACCAT GATCCTG-3' for PDGF, 5' -TGTGGGGG TGGACGGGCCGGGTAGA-3' for VEGF, and 5' -TTGGTGGTGGTGGTTGTGGTG GTGGTGG-3' for nucleolin. FAM-labeled C10 (5'-CCCCCCCCCC-3') was used as a random control DNA. Each aptamer was dropped and incubated on the designated position of a GO array, and $100 \mathrm{nM}$ of each protein was placed on a single column of the GO array.

1. Loh, K. P., Bao, Q., Eda, G. \& Chhowalla, M. Graphene oxide as a chemically tunable platform for optical applications. Nat. Chem. 2, 1015-1024 (2010).

2. Wang, Y., Li, Z., Wang, J., Li, J. \& Lin, Y. Graphene and graphene oxide: biofunctionalization and applications in biotechnology. Trends Biotechnol. 29, 205-212 (2011)

3. Morales-Narvaez, E. \& Merkoci, A. Graphene oxide as an optical biosensing platform. Adv. Mater. 24, 3298-3308 (2012).

4. Dreyer, D. R., Park, S., Bielawski, C. W. \& Ruoff, R. S. The chemistry of graphene oxide. Chem. Soc. Rev. 39, 228-240 (2010).

5. Shang, J. et al. The origin of fluorescence from graphene oxide. Sci. Rep. 2 (792), 1-8 (2012).

6. Kim, J., Cote, L. J., Kim, F. \& Huang, J. Visualizing graphene based sheets by fluorescence quenching microscopy. J. Am. Chem. Soc. 132, 260-267 (2010).

7. Wang, Y., Kurunthu, D., Scott, G. W. \& Bardeen, C. J. Fluorescence quenching in conjugated polymers blended with reduced graphitic oxide. J. Phys. Chem. C 114, 4153-4159 (2010).

8. Dong, H., Gao, W., Yan, F., Ji, H. \& Ju, H. Fluorescence resonance energy transfer between quantum dots and graphene oxide for sensing biomolecules. Anal. Chem. 82, 5511-5517 (2010)

9. Swathi, R. S. \& Sebastian, K. L. Resonance energy transfer from a dye molecule to graphene. J. Chem. Phys. 129, 054703 (2008).

10. Swathi, R. S. \& Sebastian, K. L. Long range resonance energy transfer from a dye molecule to graphene has (distance)-4 dependence. J. Chem. Phys. 130, 086101 (2009).

11. He, S. et al. A graphene nanoprobe for rapid, sensitive, and multicolor fluorescent DNA analysis. Adv. Funct. Mater. 20, 453-459 (2010).

12. Lu, C.-H., Yang, H.-H., Zhu, C.-L., Chen, X. \& Chen, G.-N. A Graphene platform for sensing biomolecules. Angew. Chem. Int. Ed. 48, 4785-4787 (2009).

13. Liu, F., Choi, J. Y. \& Seo, T. S. Graphene oxide arrays for detecting specific DNA hybridization by fluorescence resonance energy transfer. Biosens. Bioelectron. 25, 2361-2365 (2010).

14. Liu, M. et al. A graphene-based platform for single nucleotide polymorphism (SNP) genotyping. Biosens. Bioelectron. 26, 4213-4216 (2011).

15. Wu, C., Zhou, Y., Miao, X. \& Ling, L. A novel fluorescent biosensor for sequencespecific recognition of double-stranded DNA with the platform of graphene oxide. Analyst 136, 2106-2110 (2011).

16. Dong, H. et al. Highly sensitive multiple microRNA detection based on fluorescence quenching of graphene oxide and isothermal strand-displacement polymerase reaction. Anal. Chem. 84, 4587-4593 (2012).

17. Liu, X., Aizen, R., Freeman, R., Yehezkeli, O. \& Willner, I. Multiplexed aptasensors and amplified DNA sensors using functionalized graphene oxide: Application for logic gate operations. ACS Nano 6, 3553-3563 (2012).

18. Chang, H., Tang, L., Wang, Y., Jiang, J. \& Li, J. Graphene fluorescence resonance energy transfer aptasensor for the thrombin detection. Anal. Chem. 82 2341-2346 (2010)

19. Wang, X. et al. Ultrasensitive and selective detection of a prognostic indicator in early-stage cancer using graphene oxide and carbon nanotubes. Adv. Funct. Mater. 20, 3967-3971 (2010).

20. Lu, C.-H. et al. General approach for monitoring peptide-protein interactions based on graphene-peptide complex. Anal. Chem. 83, 7276-7282 (2011).

21. Wang, Y., Xiao, Y., Ma, X., Lia, N. \& Yang, X. Label-free and sensitive thrombin sensing on a molecularly grafted aptamer on graphene. Chem. Commun. 48, 738-740 (2012).

22. Chou, S. S. et al. Nanoscale graphene Oxide (nGO) as artificial receptors: implications for biomolecular interactions and sensing. J. Am. Chem. Soc. 134, 16725-16733 (2012).

23. Liang, J. et al. A highly sensitive and selective aptasensor based on graphene oxide fluorescence resonance energy transfer for the rapid determination of oncoprotein PDGF-BB. Analyst 138, 1726-1732 (2013).

24. Jang, H. et al. A graphene-based platform for the assay of duplex-DNA unwinding by helicase. Angew. Chem. Int. Ed. 49, 5703-5707 (2010). 
25. Wang, H. et al. Graphene oxide-peptide conjugate as an intracellular protease sensor for Caspase-3 activation imaging in live cells. Angew. Chem. Int. Ed. 50 7065-7069 (2011).

26. Feng, D. et al. A graphene oxide-peptide fluorescence sensor tailor-made for simple and sensitive detection of matrix metalloproteinase 2. Chem. Commun. 47, 10680-10682 (2011).

27. Lu, C.-H. et al. Multiplex detection of nucleases by a graphene-based platform J. Mater. Chem. 21, 10915-10919 (2011).

28. Li, J. et al. A graphene oxide platform for energy transfer-based detection of protease activity. Biosens. Bioelectron. 26, 3894-3899 (2011).

29. Lee, J., Kim, Y.-K. \& Min, D.-H. A new assay for endonuclease/methyltransferase activities based on graphene oxide. Anal. Chem. 83, 8906-8912 (2011).

30. Zhao, X.-H. et al. Graphene-DNAzyme Based Biosensor for Amplified Fluorescence "Turn-On" Detection of $\mathrm{Pb}^{2+}$ with a High Selectivity. Anal. Chem. 83, 5062-5066 (2011).

31. Liu, F., Ha, H. D., Han, D. J. \& Seo, T. S. Photoluminescent Graphene Oxide Microarray for Multiplex Heavy Metal Ion Analysis. Small 9, 3410-3414 (2013).

32. Huang, J., Zheng, Q., Kim, J.-K. \& Li, Z. A molecular beacon and graphene oxidebased fluorescent biosensor for $\mathrm{Cu}^{2+}$ detection. Biosens. Bioelectron. 43, 379-383 (2013).

33. Li, M., Zhou, X., Ding, W., Guo, S. \& Wu, N. Fluorescent aptamer-functionalized graphene oxide biosensor for label-free detection of mercury(II). Biosens. Bioelectron. 41, 889-893 (2013).

34. Cao, L. et al. Visual and high-throughput detection of cancer cells using a graphene oxide-based FRET aptasensing microfluidic chip. Lab Chip 12, 4864-4869 (2012).

35. Ellington, A. D. \& Szostak, J. W. In vitro selection of RNA molecules that bind specific ligands. Nature 346, 818-822 (1990).

36. Tuerk, C. \& Gold, L. Systematic Evolution of Ligands by Exponential Enrichment: RNA Ligands to Bacteriophage T4 DNA Polymerase. Science 249, 505-510 (1990).

37. Morris, K., Jensen, K., Julin, C., Weil, M. \& Gold, L. High affinity ligands from in vitro selection: Complex targets. Proc. Natl. Acad. Sci. U.S.A. 95, 2902-2907 (1998)

38. Schena, M., Shalon, D., Davis, R. W. \& Brown, P. O. Quantitative Monitoring of Gene Expression Patterns with a Complementary DNA Microarray. Science 270, 467-470 (1995).

39. Schena, M. et al. Paralle human genome analysis: Microarray-based expression monitoring of 1000 genes. Proc. Natl. Acad. Sci. U.S.A. 93, 10614-10619 (1996).

40. Sasik, R., Woelk, C. H. \& Corbeil, J. Microarray truths and consequences. J. Mol. Endocrinol. 33, 1-9 (2004).

41. Liu, M. et al. DNAzyme-based fluorescent microarray for highly selective and sensitive detection of lead(II). Analyst 137, 70-72 (2012).

42. Shalon, D., Smith, S. J. \& Brown, P. O. A DNA Microarray System for Analyzing Complex DNA Samples Using Two-color Fluorescent Probe Hybridization. Genome Res. 6, 639-645 (1996).

43. Kovtyukhova, N. I. et al. Layer-by-Layer Assembly of Ultrathin Composite Films from Micron-Sized Graphite Oxide Sheets and Polycations. Chem. Mater. 11, 771-778 (1999).
44. Lee, D. W. et al. Highly controllable transparent and conduting thin films using layer-by-layer assembly of oppositely charged reduced graphene oxides. J. Mater. Chem. 21, 3438-3442 (2011).

45. Hwang, H. et al. Highly Tunable Charge Transport in Layer-by-Layer Assembled Graphene Transistors. ACS Nano 6, 2432-2440 (2012).

46. Zhang, X., Chen, H. \& Zhang, H. Layer-by-layer assembly: from conventional to unconventional methods. Chem. Commun. 1395-1405 (2007).

47. Tang, Z., Wang, Y., Podsiadlo, P. \& Kotov, N. A. Biomedical Applications of Layer-by-Layer Assembly: From Biomimetics to Tissue Engineering. Adv. Mater. 18, 3203-3224 (2006)

48. Hummers, W. S. \& Offeman, R. E. Preparation of Graphitic Oxide. J. Am. Chem. Soc. 80, 1339-1339 (1958).

49. Li, D., Müller, M. B., Gilje, S., Kaner, R. B. \& Wallace, G. G. Processable aqueous dispersions of graphene nanosheets. Nat. Nanotechnol. 3, 101-105 (2008).

50. Mohanty, N. \& Berry, V. Graphene-Based Single-Bacterium Resolution Biodevice and DNA Transistor: Interfacing Graphene Derivatives with Nanoscale and Microscale Biocomponents. Nano Lett. 8, 4469-4476 (2008).

51. Husale, S. et al. ssDNA Binding Reveals the Atomic Structure of Graphene. Langmuir 26, 18078-18082 (2010).

\section{Acknowledgments}

This research was supported by Basic Science Research Program through the National Research Foundation of Korea (NRF) funded by the Ministry of Education, Science and Technology (NRF-2012R1A1A3015259) and by the grant funded by the Korean Government (No. 2010-0028684)

\section{Author contributions}

Y.K.J. and B.-S.K. designed the research. Y.K.J. performed all experiments. T.L. and E.S contributed to testing the reproducibility of the data. Y.K.J. and B.-S.K. contributed to analysis and wrote the manuscript and supporting text. All authors reviewed the manuscript.

\section{Additional information}

Supplementary information accompanies this paper at http://www.nature.com/ scientificreports

Competing financial interests: The authors declare no competing financial interests.

How to cite this article: Jung, Y.K., Lee, T., Shin, E. \& Kim, B.-S. Highly Tunable Aptasensing Microarrays with Graphene Oxide Multilayers. Sci. Rep. 3, 3367; DOI:10.1038/ srep03367 (2013)

c) (i) $\Theta$ This work is licensed under a visit http://creativecommons.org/licenses/by-nc-nd/3.0 